\title{
¿Conviene seguir fomentando las plantaciones forestales en el norte de la Patagonia Argentina? ¿Dónde? ¿Para qué? ¿A quién le conviene?
}

\author{
José PARUelo \\ Laboratorio de Análisis Regional y Teledetección y Departamento de Métodos Cuantitativos y Sistemas de Información. IFEVA \\ y Facultad de Agronomía - CONICET y UBA. Ciudad Autónoma de Buenos Aires, Argentina. IECA-Maestría en Ciencias \\ Ambientales. Facultad de Ciencias. Universidad de la República. URUGUAY.
}

\begin{abstract}
Resumen. El objetivo de este artículo es plantear algunos de los aspectos que deberían ser tenidos en cuenta a la hora de decidir el fomento de plantaciones forestales en el noroeste de la Patagonia y discutir cómo puede contribuir el sistema de Ciencia y Técnica (SCyT) al proceso de toma de decisiones en el marco de un proceso de Ordenamiento Territorial. Particularmente se discute el papel de la Ciencia y los científicos en: a) el reconocimiento de la heterogeneidad del sistema territorial y su caracterización, tanto en los aspectos humanos como biofísicos (por ej. desde comunidades vegetales a asociaciones de productores), b) la definición de la aptitud de distintas unidades del territorio para la actividad forestal y la evaluación de los impactos (absolutos y relativos a otras alternativas) de esas actividades sobre distintas dimensiones (ambiental, social y económica), c) la definición del "problema", o sea la construcción social a partir de situaciones que distintos actores valoran de manera diferente (por ej. establecer una plantación de pinos). La construcción de "árboles" de causas y efectos, por un lado, y el análisis de los cambios en la provisión de Servicios Ecosistémicos (SE) y de quienes se apropian de sus beneficios, por otro, son caminos a través de los cuales el SCyT puede contribuir al proceso de toma de decisiones asociado a la actividad forestal en Patagonia.
\end{abstract}

[Palabras clave: ordenamiento territorial, árbol de problemas, sistemas socio-ecológicos, actores, desertificación, estepas patagónicas]

\begin{abstract}
AвSTRACT. Is it advisable to further promote forest plantations in northern Patagonia argentina? Where, why and for whom?: The objective of this article is to discuss: 1) some of the issues to be considered to decide the promotion of pine plantations in Patagonia, and 2) how the scientific community may contribute to the decision making processes. Particularly, the article discusses the role of Science and scientists on: a) the description of the heterogeneity of the territory, both in the biophysical and human dimensions, b) the definition of the sylvicultural aptitude of the different land units and the evaluation of the impacts of afforestation (absolute and relative to other activities) on the environmental, social and economics dimensions, c) the definition of the "problem" (i.e., the social construction from a set of situations that stakeholders value differentially). The construction of "trees" of effects and impacts, the analysis of the changes in the provision of Ecosystem Services and the distribution of benefits among different stakeholders are some of the potential contributions of the scientific community to the decision making processes on afforestation in Patagonia.
\end{abstract}

[Keywords: land use planning, problem tree method, socioecological systems, actors, desertification, Patagonian steppes]

\section{INTRODUCCIÓN}

Empecemos por no generar expectativas: en este artículo no voy responder el interrogante planteado por los editores de este Foro-Debate, es decir, si conviene seguir fomentando las plantaciones forestales en el norte de la Patagonia argentina. Mi objetivo es plantear algunos de los aspectos que deberían ser tenidos en cuenta a la hora de dar respuesta a esta pregunta y discutir cómo puede contribuir el sistema de Ciencia y Técnica (SCyT). Mi perspectiva surge de la experiencia en estudios de caracterización y manejo de áreas de estepa y del ecotono entre la estepa y el bosque en el área. Esta perspectiva define un sesgo en la presentación de ejemplos pero no necesariamente restringe el alcance de las afirmaciones que presento. Numerosos trabajos han tratado los aspectos silviculturales o los efectos de las plantaciones (Gonda 1998; Laclau et al. 2002; Laclau 2003, 2005; Fernández et al. 2008, 2009; Gyenge et al. 2002, 2005, 2008, 2009; Licata et al. 2008). Remito al lector a ellos para detalles.

Las políticas de fomento de plantaciones forestales son una de las herramientas de mayor impacto en procesos de ordenamiento del territorio (OT). Las consecuencias de las forestaciones deben ser entonces pensadas en el contexto del territorio o del sistema 
socio-ecológico sobre el cual impactarán y como parte de políticas de OT, aún cuando no exista una mención explícita a este proceso. El OT se concibe como un proceso político - técnico - administrativo orientado a la organización, planificación y gestión del uso y ocupación del territorio, en función de las características y restricciones biofísicas, culturales, socioeconómicas y políticoinstitucionales. Este proceso debe basarse en objetivos explícitos. En comparación con otras aproximaciones, propicia un uso más inteligente y justo del territorio, aprovecha oportunidades, reduce riesgos, protege recursos en el largo plazo y reparte en forma racional costos y beneficios del uso y la conservación entre los actores involucrados (Paruelo et al. 2014).

Partamos de dos supuestos para explorar las preguntas del título. Primero, si la promoción de las forestaciones está en discusión es porque hay un conflicto en torno a ellas (explícito o implícito, presente o potencial). En otros términos, el evento "promover las forestaciones" es percibido de manera positiva, neutra o negativa por distintos actores sociales. La actitud hacia las forestaciones resulta de una serie factores. Algunos de ellos están ligados a intereses (explícitos o no) de distintos actores. En última instancia, se vinculan con una distribución desigual entre los distintos actores de los costos y beneficios (sociales, ambientales y económicos) asociados a la actividad. Otros factores se asocian a la falta de información o a información errónea o sesgada acerca de esos costos y beneficios. Por último hay factores relacionados a cuestiones ideológicas, es decir a valores que llevan a algunos actores a priorizar la rentabilidad a la conservación de una especie y a otros a lo contrario. Intereses, información y valores son aspectos centrales a considerar desde la perspectiva que asumo para tratar el tema.

Un segundo supuesto es que las decisiones acerca de la promoción o no de plantaciones forestales se basan en un proceso de planificación participativo en el que las asimetrías de poder entre los distintos actores serán balanceadas por el Estado. Si las decisiones ocurriesen a través de otros procesos (por ej. decisiones individuales de privados o de agencias gubernamentales), buena parte de las argumentaciones que presento más abajo serían irrelevantes. Claramente la verosimilitud de este supuesto está ligada a qué grupos sociales y políticos ejerzan el control del Estado.

Las contribuciones del SCyT al proceso de planificación y toma de decisiones pueden ser muy variadas y ligadas a uno de los factores que mencionaba más arriba: la información, desde su acceso a su confiabilidad. Por un lado, se asocian al reconocimiento de la heterogeneidad del sistema territorial y a su caracterización. La caracterización de la heterogeneidad involucra tanto los aspectos humanos como biofísicos (desde comunidades vegetales a asociaciones de productores) e incluye la identificación de patrones espaciales y temporales. Por otro lado, y una vez caracterizada la heterogeneidad, el SCyT puede ayudar en la definición de la aptitud de distintas unidades del territorio para la actividad forestal, a la evaluación de la sustentabilidad y de los impactos (absolutos y relativos a otras alternativas) de esas actividades en sus distintas dimensiones relevantes (ambiental, social y económica).

La posibilidad de que el SCyT contribuya a la toma de decisiones depende de una clara definición del "problema". Lo que denominamos problema es una construcción social a partir de situaciones que distintos actores valoran de manera diferente, por ej., establecer una plantación de Pinus ponderosa en El Maitén, Chubut. Los "árboles de problemas" (Ortegón et al. 2005, Román 2012), que permiten identificar factores causales y efectos, son una herramienta muy poderosa en la solución de conflictos al hacer explícitas las hipótesis acerca de los beneficios y perjuicios de lo que se identifica como problema (o situación generadora de conflictos) y de los factores que lo determinan. Más aún, los esquemas de este tipo, permiten hacer explícitas las evidencias empíricas y/o la secuencia lógica en las que se apoyan dichas hipótesis. El SCyT puede, además, jugar el papel que Pielke (2007) denomina "promotor de alternativas". Frente a situaciones de disputa y de trabas en la decisión ("Sí vs. No a las forestaciones"), el sistema puede aportar visiones alternativas de usos del territorio que no estaban consideradas originalmente.

Sin embargo una serie de factores complican un abordaje "aséptico" desde el SCyT. Estos incluyen, como mínimo: disputas de valores e intereses, asimetrías de poder y en el acceso a la información; distintas percepciones tanto a escalas temporales y espaciales como de los 
actores involucrados. Incorporar a la Ciencia y a los científicos permite trabajar sobre algunos de estos factores, pero no sobre otros, especialmente las disputas de valores e intereses y las asimetrías de poder.

\section{RECONOCER HETEROGENEIDAD}

Para que el SCyT pueda ayudar a identificar el problema, evaluar aptitudes e impactos y promover alternativas es necesario, como primer paso ineludible, describir y caracterizar la heterogeneidad del sistema socio-ecológico a tratar. Esta descripción debe necesariamente incluir las dimensiones humanas y biofísicas del sistema.

La dimensión humana: Como ya lo adelantamos, si hay discusión en torno de una intervención en el territorio es porque hay un conflicto manifiesto o potencial. Eso implica, que no a todos los actores les conviene o agrada forestar. Los actores tienen que ser identificados y caracterizados en cuanto a su posición respecto del tema (Freeman, 1984) y de su poder o influencia (Tapella 2007). Esta caracterización de los actores permite identificar qué actores se apropian de los distintos beneficios y perjuicios derivados de la expansión de la actividad forestal. Esta caracterización nos acerca a la respuesta de una de las preguntas planteadas en el título: ¿a quién le conviene? En última instancia, ¿de dónde salen los recursos para realizar las forestaciones?, ¿cómo se distribuyen los beneficios?, ¿quién percibe los perjuicios?

La dimensión biofísica: La caracterización de la heterogeneidad tendrá que tener en cuenta la aptitud de las distintas unidades del territorio para la actividad forestal. Esto será función, fundamentalmente, de la precipitación y de propiedades del suelo (especialmente su pendiente, textura y profundidad).

Como señalábamos antes, el otro componente importante a considerar es el impacto. Éste dependerá, entre otras cosas, de qué es lo que se reemplaza. En esto se debe considerar por un lado el tipo de vegetación presente (bosque caducifolio, bosque de coníferas, matorral, estepa graminosa, estepa arbustiva, etc.) y, por otro, el nivel de alteración del tipo de vegetación original. En Patagonia, la aptitud y los impactos varían de manera fundamental entre las dos grandes unidades biogeográficas que son los bosques subantárticos y las estepas patagónicas (Paruelo et al. 2007). Cada una de estas unidades presenta a su vez diferencias internas muy marcadas asociada a variaciones en la disponibilidad de recursos (León et al 1998).

Un componente adicional a considerar en la caracterización de la vegetación que eventualmente reemplazaría una forestación es su grado de deterioro. Numerosos trabajos muestran que el pastoreo ovino y vacuno genera cambios estructurales y funcionales importantes en las estepas y en el ecotono con el bosque (Soriano 1956, León y Aguiar 1985, Paruelo y Aguiar 2003), asociados a un proceso de desertificación (Verón et al. 2006, Verón y Paruelo, 2010). Las modificaciones derivadas del pastoreo implican cambios fisonómicos (estepas graminosas que se transforman en semidesiertos, Paruelo, 2005), funcionales (cambios en la productividad, Verón y Paruelo, 2010) o en el suelo (pérdida de suelo por erosión y formación de pavimentos de erosión, Movia y Soriano1986). La reversibilidad de algunos de los estados de deterioro, particularmente de aquellos que involucran la pérdida de suelo, es muy baja (Paruelo et al. 1993).

Finalmente la caracterización de la estructura, composición y configuración de las cuencas permite incorporar la perspectiva del paisaje en la descripción de la heterogeneidad. Esto incluye, además de las características morfométricas de las cuencas, la proporción y distribución de las distintas unidades en el espacio. La perspectiva del paisaje es esencial en una planificación que busque distribuir beneficios y perjuicios de las actividades forestales.

\section{LA UTILIDAD DE LOS ÁRBOLES DE PROBLEMAS}

Un árbol de problemas o de causas y efectos para la zona del distrito subandino de la estepa patagónica y del ecotono con los bosques subantárticos (León et al. 1998) permite explicitar los factores que estarían operando en determinar cambios en la superficie implantada con especies forestales (Figura 1). Las hipótesis planteadas en el árbol deben ser fundadas en cuanto a su base empírica (estudios locales, regionales o de otras áreas similares), en su fundamento teórico y en su estructura lógica. Más aún (y a diferencia del ejemplo presentado en la Figura 1), el árbol de problemas debería resultar de un proceso 


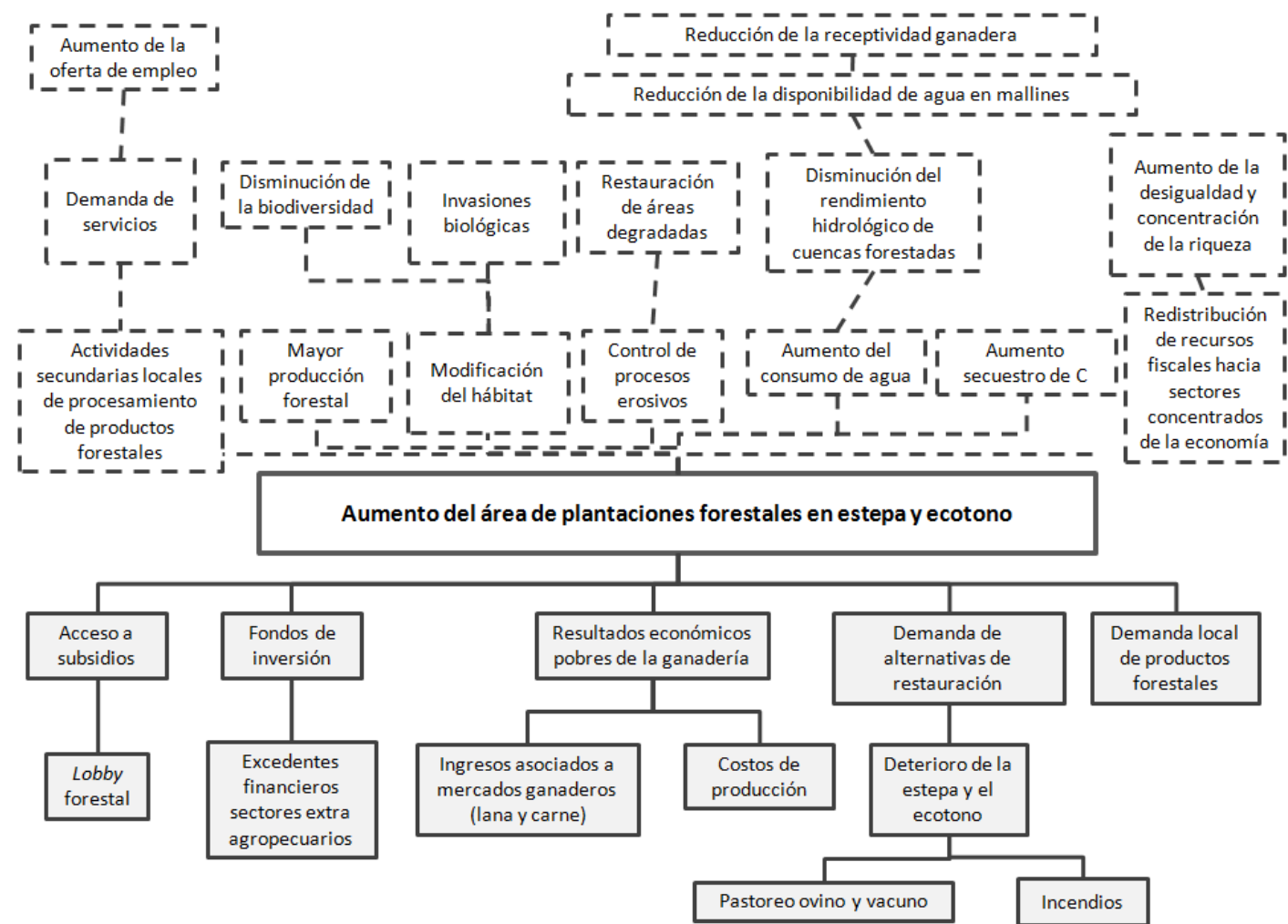

Figura 1. "Arbol de problemas" asociados al aumento del área de plantaciones forestales en el ecotono entre la estepa y el bosque en la Patagonia Argentina. Las cajas grises y con bordes de líneas llenas corresponden a las hipótesis de causas de aumento de la forestación y las vacías con bordes de líneas punteadas a los efectos de esos cambios.

Figure 1. Scheme of causes and effects ("Problem tree") associated to the increase of the afforested area in the foreststeppe ecotone of the Argentine Patagonia. Grey boxes with solid lines correspond to the hypothetical causes of afforestaion increase and the dotted lines boxes to the effects of these changes.

participativo en donde los diferentes actores pueden volcar sus hipótesis acerca de qué factores operan, qué consecuencias se generan y qué relaciones existen entre esos factores. La elaboración del árbol de problemas, como la construcción de cualquier modelo, permite organizar el conocimiento e identificar relaciones causales con distinto grado de apoyo empírico. Por ejemplo, para la rama de efectos asociados a la dinámica del agua, las hipótesis específicas podrían apoyarse en estudios de evapotranspiración basados en sensores remotos y de drenaje profundo derivados de modelos de simulación (Mylkovic 2011). Esos estudios muestran que las plantaciones de pino evapotranspirarían en promedio un $64 \%$ más que las estepas arbustivo-graminosas que reemplazan, y que esos niveles de evapotranspiración determinarían un drenaje profundo nulo en forestaciones y una merma en el escurrimiento subsuperficial que llegaría a los mallines. Por supuesto, la magnitud de esa reducción dependerá de la proporción forestada de las cuencas y de su configuración.
La rama del árbol de efectos relacionada al eventual secuestro de carbono por parte de las plantaciones también cuenta con apoyo empírico. Nosetto et al. (2006) muestran que luego de 15 años las forestaciones en el $\mathrm{N}$ de Chubut y en áreas con menos de 500 mm de precipitación al año, agregan carbono al sistema, con tasas de secuestro del orden de $0.5-3.3 \mathrm{Mg} \mathrm{C}$ ha $^{-1} \mathrm{año}^{-1}$. Las ganancias de carbono fueron mayores en las estepas degradas en donde se implantaron los pinos, tanto en la parte aérea como subterránea $(+150 \%$ y $+32 \%)$. Las diferencias estuvieron asociadas a la biomasa y no se observaron diferencias en el carbono orgánico del suelo.

Además de explicitar hipótesis y documentar su apoyo empírico, el modelo del árbol de problemas tiene otras virtudes. Al identificar la diversidad de efectos de la situación problemática, el árbol de problemas ayuda a definir cuál de esos efectos específicos es particularmente resistido o valorado por los distintos actores. Eso abre posibilidades concretas de negociación. Si el principal efecto 
no deseado es la disminución del drenaje subsuperficial en mallines, las forestaciones sí podrían realizarse en cuencas cuyas partes bajas no presenten mallines. En cambio si la preocupación principal deriva del riesgo de que se generen condiciones favorables para especies invasoras o que se destinen fondos públicos a subsidiar actividades cuyos beneficios son de apropiación privada, las forestaciones no tendrían cabida en ninguna cuenca.

Un árbol de causas y efectos es la base de la definición de objetivos de intervención en el territorio. Hacer explícitos los supuestos permite definir cuáles de ellos promoverán y cuales se buscará evitar o revertir. Dependiendo de esto la promoción de las plantaciones puede plantearse como: (i) Como terapia de remediación, cuando el sistema está degradado y la probabilidad de recuperación se considera muy baja. (ii) Para satisfacer necesidades, cuando existe una demanda local de productos forestales, y/o se quiere fomentar el empleo en ese sector. (iii) Para apropiarse de una renta y / o recursos financieros, cuando existe la oportunidad de acceder a recursos fiscales o se accede a mercados externos al territorio. Estos objetivos serán valorados de manera diferente por los distintos actores involucrados según sus intereses y valores.

\section{ANÁLISIS DE LA PROVISIÓN DE SERVICIOS ECOSISTÉMICOS Y SU APROPIACIÓN}

Una manera de evaluar la conveniencia o no de una forestación es en términos de los cambios en el nivel de provisión de Servicios Ecosistémicos (SE) (Paruelo et al. 2014). Esto requiere: a) identificar los SE clave, b) cuantificar el cambio en el nivel de provisión en base a la superficie y configuración de las forestaciones en la cuenca o el paisaje, c) definir los niveles de pérdida de esos SE que se tolera perder o resignar, y en qué medida el aumento de otros compensa la pérdida.

Identificar cuáles de los múltiples servicios serán priorizados es uno de los puntos críticos en este proceso. De hecho es un subproducto de la elaboración del árbol de causas y efectos (Figura 1). Varias de las cajas (secuestro de carbono, producción de commodities, calidad del hábitat, dinámica del agua) son ejemplos de esos SE. Los SE clave en principio serán aquellos más vulnerables al cambio y que tengan las menores opciones tecnológicas o ecológicas para su sustitución. Si bien la definición de los SE a considerar involucra aspectos técnicos, cuáles de ellos se priorizarán es una decisión eminentemente política. Detrás de la decisión de considerar de manera prioritaria a la provisión de agua potable, la regulación de inundaciones, el secuestro de carbono, la conservación de determinada especie o la producción de forraje existe una puja de intereses y valores que suele dirimirse en función del poder acumulado por los distintos actores.

Uno de los pre-requisitos para darle racionalidad al proceso de priorización de SE (es decir, capacidad de alcanzar objetivos comunes explícitos) consiste en la capacitación e información de los actores. La discusión de prioridades debe ir precedida de la instalación del marco conceptual de los SE en el sistema socio-ecológico. Esto implica desde un curso de capacitación para personas clave en cada grupo de actores, hasta campañas de educación y divulgación capaces de instalar el enfoque en los diversos sectores de la sociedad. Incluye también la discusión de la idea de compromiso (en el sentido de trade-off) en la provisión de distintos SE y el planteo de alternativas de valoración para los distintos SE.

Una vez definidos los SE, el siguiente paso es describir cómo un aumento de la superficie forestada de la cuenca modifica la provisión de cada uno de los SE. Esa relación o función de afectación o impacto de un SE define la manera según la cual cambia el nivel de provisión ante cambios de un factor de estrés o perturbación del sistema original o de referencia (la estepa). La función de impacto tiene una escala asociada, la del paisaje en consideración. Así los factores de estrés y perturbación serán caracterizados muchas veces como porcentaje de transformación o alteración del paisaje o, su complemento, el porcentaje de hábitat natural. Para el caso del área de forestación como factor de estrés o perturbación existirá una familia de funciones que tomen en cuenta la diferencia en la configuración de la cuenca, la densidad de plantación, la posición en el gradiente de precipitaciones de la cuenca, y el grado de deterioro de la vegetación que se reemplaza. Un ejemplo de estas funciones de afectación es la que presenta Milkovic (2011). En base a estimaciones de evapotranspiración derivadas de datos espectrales y de modelos de 
simulación en ese trabajo se describe el cambio esperado en el rendimiento hidrológico de una cuenca en función del área forestada. Para el caso de áreas en donde llueven menos de 400 $\mathrm{mm}$ anuales, con el 5\% del área cubierta por mallines, y considerando valores medios de percolación de la estepa y de la forestación de $30 \%$ y $0.5 \%$ respectivamente, el rendimiento hidrológico se reduce a menos del 30\% del original cuando la superficie forestada de la cuenca supera el $65 \%$. Como señalábamos, estas funciones cambiarán si la precipitación es mayor o la vegetación original cubre menos el suelo. En Patagonia, en sitios con precipitación media anual de 800 y $1200 \mathrm{~mm}$, no se han detectado cambios en la productividad ni en el nivel de la napa en mallines ubicados próximos a forestaciones de pinos de distintas densidades (Weigandt et al. 2011). El aumento en el drenaje profundo y de la escorrentía superficial de áreas degradas de la cuenca (Parizek et al. 2002) compensaría la merma del rendimiento hidrológico causada por las plantaciones.

\section{CONSIDERACIONES FINALES}

Buscar en el SCyT una respuesta a la pregunta " ¿Conviene seguir fomentando las plantaciones forestales en el norte de la Patagonia Argentina?" es, en mi opinión, un error o un intento de transformar a los investigadores en abogados de una causa (issue advocates, o activistas según la clasificación de roles de los científicos que propone Pielke (2007)). En la respuesta a esa pregunta pesan fundamentalmente valores, intereses, el contexto socio-político-cultural, etc. ¿Eso quiere decir que los científicos que trabajan en el área y en esos temas no tienen ningún papel? De ninguna manera. Sin embargo, el papel sería otro: proveer el marco conceptual en el cual discutir el tema y las evidencias empíricas que sustentan la toma de decisiones. Esto incluye contribuir a definir "el problema", fundamentalmente los factores (causas) que determinan esa situación problemática (la forestación en la estepa) y sus consecuencias en distintas dimensiones. El árbol de problemas, la cuantificación de cambios en el nivel de provisión de servicios ecosistémicos clave y el análisis de la apropiación diferencial por parte de los distintos actores de costos y beneficios es uno de los caminos para ayudar a que la sociedad dé respuesta a la pregunta formulada en el título y a muchas otras similares.
Agradecimientos: A Marcela Román y a los estudiantes del curso de Ordenamiento Territorial de la Lic. en Ciencias Ambientales que me mostraron las virtudes del árbol de problemas. A Luciana Staiano, Juliana Mossi, María Vallejos y Agustina García Collazo por sus sugerencias y aportes. Este trabajo se llevó a cabo con la financiación del proyecto del Inter-American Institutefor Global Change Research (IAI) CRN3095 el cual a su vez es financiado por la US NationalScienceFoundation (Grant GEO-1128040) y con el aporte de proyectos FONCYT, UBACYT y PIP CONICET

\section{BiBLIOGRAFÍA}

FERNÁNDEZ, ME; J GYenge; J LiCATA; T SCHLICHTER \& BJ BOND. 2008. Belowground interactions for water between trees and grasses in a temperate semiarid agroforestry system. Agroforestry Systems, 74(2):185-197.

FERnÁNDEZ, ME; J GYenge \& T SChlichter. 2009. Water flux and canopy conductance of natural versus planted forests in Patagonia, South America. Trees, 23(2):415427.

FreEmAn, RE. 1984. Strategic Management: A Stakeholder Approach. Pitman Series in Business and Public Policy. Publisher: Harpercollins College Div. $1^{\text {st }}$ ed. Pp. 275.

GonDA, HE. 1998. Height-diameter and volume equations, growth intercept and needle length site quality indicators, and yield equations for Young Ponderosa Pine Plantations in Neuquén, Patagonia, Argentina. Tesis de PhD. College of Forestry, Forest Resources Department, Oregon State University, USA.

Gyenge, JE; ME Fernández; G Dalla Salda \& TM SCHLICHTER. 2002. Silvopastoral systems in Northwestern Patagonia II: water balance and water potential in a stand of Pinus ponderosa and native grassland. Agroforestry Systems, 55(1):47-55.

GyenGe,JE; ME FERNÁNDEZ; G DALla SALDA \& TMSChlichter. 2005. Leaf and whole-plant water relations of the Patagonian conifer Austrocedrus chilensis (D. Don) Pic. Ser. et Bizzarri: implications on its drought resistance capacity. Annals of Forest Science, 62(4):297-302.

GYENGE, J; ME FERnÁNDEZ; M SARASOla \& T SCHLICHTER. 2008. Testing a hypothesis of the relationship between productivity and water use efficiency in Patagonian forests with native and exotic species. Forest Ecology and Management, 255(8):3281-3287.

GYENGE, JE; ME FERNÁNDEZ \& TM SCHLICHTER. 2009. Effect of pruning on branch production and water relations in widely spaced ponderosa pines. Agroforestry Systems, 77(3):223-235.

LACLAU, P. 2002. La forestación en la Patagonia y el cambio climático. Publicaciones de Economía Forestal, Instituto Nacional de Tecnología Agropecuaria (INTA)-Agencia Alemana de Cooperación Técnica (GTZ). Bariloche, Argentina.

LACLAU, P. 2003. Biomass and carbon sequestration of ponderosa pine plantations and native cypress forests in northwest Patagonia. Forest Ecology And Management, 180(1):317-333.

LACLAU, P. 2005. Cambio climático y forestación en el noroeste de la Patagonia. Idia XXI. Revista de Información Sobre Investigación y Desarrollo Agropecuario, 5(8):262-265.

LEÓN, RJC \& MR AGUIAR. 1985. El deterioro por uso pasturil en estepas herbáceas patagónicas. Phytocoenologia, 13(2): 181-196. 
León, RJ; D Bran; M Collantes; JM Paruelo \& A Soriano. 1998. Grandes unidades de vegetación de la Patagonia extra andina. Ecología Austral, 8(2):125-144.

LicAtA, JA; JE GYenge; ME FernÁndez; T SChlichter \& BJ BOND. 2008. Increased water use by ponderosa pine plantations in northwestern Patagonia, Argentina compared with native forest vegetation. Forest Ecology and Management, 255(3):753-764.

Milkovic, M. 2011. Consecuencias del cambio en el uso del suelo sobre la dinámica del agua en dos sistemas semiáridos. Tesis de Maestría en Recursos Naturales. Escuela para Graduados "Ing. Agr. Alberto Soriano", Facultad de Agronomía, UBA.

Nosetto, MD; EG Jobbágy \& JM Paruelo. 2006. Carbon sequestration in semiarid rangelands: Comparison of Pinus ponderosa plantations and grazing exclusion in NW Patagonia. Journal of Arid Environments, 67: 142-156.

Ortegón, E \& A Prieto. 2005. Metodología del marco lógico para la planificación, el seguimiento y la evaluación de proyectos y programas. Vol. 42. United Nations Publications.

ParizeK, B; CM Rostagno \& R Sottini. 2002. Soil erosion as affected by shrub encroachment in north-eastern Patagonia. Journal of Range Management, 55(1):43-48.

Paruelo, JM; M Bertiller; T Schlichter \& FR Coronato. 1993. Secuencias de deterioro en distintos ambientes patagónicos: su caracterización mediante el modelo de estados y transiciones. Documento LUDEPA. INTAFAUBA-GTZ

Paruelo, JM \& MR Aguiar. 2003. Impacto humano sobre los ecosistemas. El caso de la desertificación. Ciencia Hoy, 13:48-59.

Paruelo, JM. 2005. ¿Cuánto se han desertificado las estepas patagónicas? Evidencias a partir de la memoria del sistema. Pp. 303-318 en: Oesterheld, M; MR Aguiar; C Ghersa \& JM Paruelo (eds.). La heterogeneidad de la vegetación de los agroecosistemas. Editorial Facultad de Agronomía (Buenos Aires).

Paruelo, JM; EG Jobbágy; M Oesterheld; RA Golluscio \& MR AGUiar. 2007. The grasslands and steppes of Patagonia and the Rio de la Plata plains. Chapter 14. Pp 232-248 en: Veblen, T; K Young \& A Orme (eds.). The Physical Geography of South America. The Oxford Regional Environments Series, Oxford University Press.

Paruelo, JM; EG Jobbágy; P Laterra; H Diéguez; MA García CollazO; ET AL. (EDS.). Ordenamiento Territorial: Conceptos, Metodologías y Experiencias. FAO/ MAG/ UBA. 2014.

PIELKE, RA. 2007. The honest broker: Making sense of science in policy and politics. Cambridge University Press. Cambridge, UK.

Román, M. 2012. Diseño y Evaluación Financiera de Proyectos Agropecuarios. Editorial Facultad de Agronomía (EFA). Universidad de Buenos Aires. Pp. 126.

SORIANO, A. 1956. Aspectos ecológicos y pasturiles de la vegetación patagónica relacionados con su estado y capacidad de recuperación. Revista de Investigaciones Agrícolas, 10(4):349-372.

SORIANO, A \& CP MOVIA. 1986. Erosión y desertización en la Patagonia. Interciencia, 11:77-83.

TApella, E. 2007. El mapeo de Actores Claves. Universidad Nacional de Córdoba. Inter-American Institute for Global ChangeResearch (IAI).

Verón, SR; JM PARUelo \& M Oesterheld. 2006. Assessing Desertification. Journal of Arid Environments, 66:751-763.

VERÓN, SR \& JM PARUELO. 2010. Desertification alters the response of vegetation to changes in precipitation. Journal of Applied Ecology, 47(6):1233-1241.

Weigandt, M; J Gyenge; ME Fernández; S VARELA \& T SCHLICHTER. 2011. Is forage productivity of wetlands influenced by the afforestation of upstream hillsides? A study in wetlands of NW Patagonia. Forest Systems, 20:165-175. 